\title{
Performance Analysis of Differential Beamforming in Decentralized Networks
}

\author{
Samer Alabed \\ Department of Electrical Engineering, American University of the Middle East, Kuwait.
}

\begin{tabular}{l} 
Article Info \\
\hline Article history: \\
Received Sep 16, 2017 \\
Revised Feb 18, 2018 \\
Accepted Mar 13, 2018 \\
\hline Keyword: \\
Distributed beamforming \\
Two-way relay networks \\
Distributed space-time coding \\
Differential space-time coding \\
Cooperative communications
\end{tabular}

\begin{abstract}
This paper proposes and analyzes a novel differential distributed beamforming strategy for decentralized two-way relay networks. In our strategy, the phases of the received signals at all relays are synchronized without requiring channel feedback or training symbols. Bit error rate (BER) expressions of the proposed strategy are provided for coherent and differential M-PSK modulation. Upper bounds, lower bounds, and simple approximations of the BER are also derived. Based on the theoretical and simulated BER performance, the proposed strategy offers a high system performance and low decoding complexity and delay without requiring channel state information at any transmitting or receiving antenna. Furthermore, the simple approximation of the BER upper bound shows that the proposed strategy enjoys the full diversity gain which is equal to the number of transmitting antennas.
\end{abstract}

Copyright (c) 2018 Institute of Advanced Engineering and Science. All rights reserved.

\section{Corresponding Author:}

Name: Samer Alabed

Affiliation: Assistant professor

Address: Department of Electrical Engineering, American University of the Middle East, Block 3, Building 1, Egaila, Kuwait.

Phone: +965 22251400 Ext.: 1790

Email: samer.al-abed@aum.edu.kw, samer.alabed@nt.tu-darmstadt.de

\section{INTRODUCTION}

Depending on the availability of channel state information (CSI) on the relay nodes, several strategies for wireless sensor networks have been recently suggested to generalize and improve cooperative diversity strategies [1-15]. Some strategies are based on unrealistic assumptions such as perfect CSI available at all nodes [15]. These popular strategies, such as distributed beamforming strategy utilized in wireless sensor networks, exploit perfect CSI to coherently process the relay signals. These strategies enjoy a high system performance and low decoding complexity and delay. Other strategies, such as the distributed space-time coding (DSTC), consider the case of perfect or partial CSI available at the receiving antenna only [6-8].

Recent strategies for wireless sensor networks, such as differential distributed space-time coding (DiffDSTC) strategies [6-14], have been recently designed based on more practical assumption of no CSI required to decode the information symbols. Therefore, these strategies do not associated with any overhead involved in channel estimation. However, Diff-DSTC strategies suffer from low system performance in terms of bit error rate and a comparably high decoding complexity and latency. In [16], another differential approaches based on beamforming strategies, i.e., differential receive beamforming strategies, have been suggested which do not need CSI at any transmitting or receiving antenna to decode the information symbols by combining the differential diversity strategy and the receive beamforming strategy. However, these strategies require $(R+1)$ time slots to transmit each information symbol where $R$ is the number of relay nodes. Therefore, they suffer from low spectral efficiency for a large number of relay nodes, i.e., their symbol rate is lower than that of the conventional transmit beamforming. In this paper, we propose and analyze a novel decentralized beamforming-based noncoherent relaying strategy. Our strategy can be viewed as a non-trivial combination of two multiple antenna strategies, i.e., the differential diversity strategy and the distributed beamforming strategy, in which the benefits of both strategies $[9,10]$ are retained. 


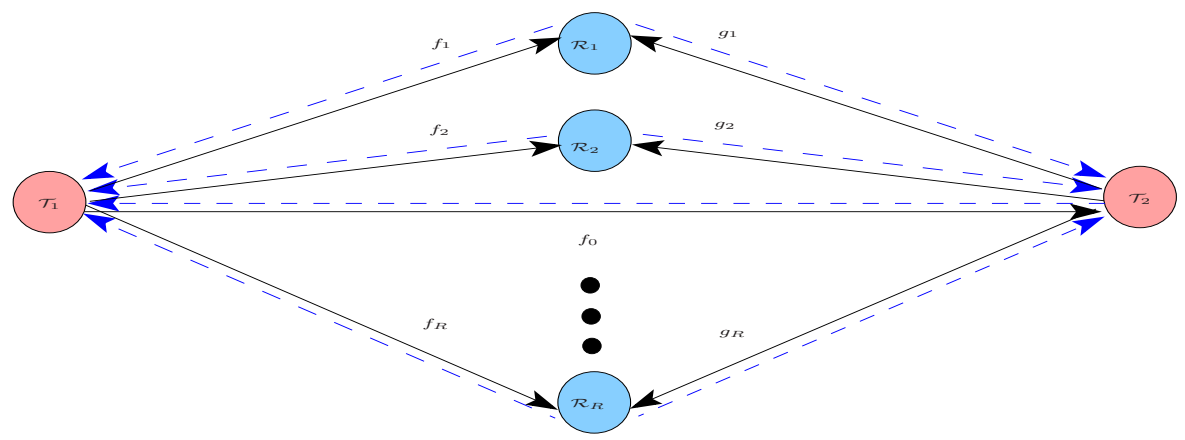

Figure 1. TWRN with $R+2$ nodes.

\section{WIRELESS RELAY NETWORK MODEL}

Let us consider a half-duplex two-way relay network consisting of two single-antenna terminals $\mathcal{T}_{1}$ and $\mathcal{T}_{2}$ that exchange their information via $R$ single-antenna relays, i.e., $\mathcal{R}_{1}, \ldots, \mathcal{R}_{R}$ as shown in Fig. 1 . We assumed that the channels are reciprocal for the transmission from terminal $\mathcal{T}_{1}$ to terminal $\mathcal{T}_{2}$ and vice versa. We further consider the extended block fading channel model, for which the channels do not change within two consecutive transmission blocks and then change randomly outside this time interval. Further, we consider the case of no CSI available at any transmitting or receiving antenna in the whole network and the nodes $\mathcal{T}_{1}, \mathcal{T}_{2}, \mathcal{R}_{1}, \ldots, \mathcal{R}_{R}$ have limited average transmit powers $P_{\mathcal{T}_{1}}, P_{\mathcal{T}_{2}}, P_{1}, \ldots, P_{R}$, respectively. Throughout this paper, $|\cdot|, \measuredangle,(\cdot)^{*},\|\cdot\|$, and $\mathrm{E}\{\cdot\}$ denote the absolute value, the argument of a complex number, the complex conjugate, the Frobenious norm, and the statistical expectation, respectively.

\section{THE OPTIMAL DECENTRALIZED COHERENT BEAMFORMING WITH PERFECT CSI}

Let us consider the case where all channel coefficients and the statistics of the noise processes at the relays and terminals are perfectly known at any time. In this part, the encoded symbols are identical with the corresponding information symbols, such that

$$
x_{\mathcal{T}_{t}}^{(k)}=s_{\mathcal{T}_{t}}^{(k)}
$$

where $s_{\mathcal{T}_{t}}^{(k)}$ denotes the information symbol of the $k$ th block transmitted by terminal $\mathcal{T}_{t}$ and $t=1,2$. During the first and second time slot of the $k$ th transmission block, the $r$ th relay receives from $\mathcal{T}_{1}$ and $\mathcal{T}_{2}$ the following signals

$$
\begin{aligned}
& y_{\mathcal{R} 1, r}^{(k)}=\sqrt{P_{\mathcal{T}_{1}}} f_{r}^{(k)} x_{\mathcal{T}_{1}}^{(k)}+n_{\mathcal{R} 1, r}^{(k)} \\
& y_{\mathcal{R} 2, r}^{(k)}=\sqrt{P_{\mathcal{T}_{2}}} g_{r}^{(k)} x_{\mathcal{T}_{2}}^{(k)}+n_{\mathcal{R} 2, r}^{(k)}
\end{aligned}
$$

where $n_{\mathcal{R} t, r}^{(k)}$ denotes the noise signal at the $r$ th relay in the $t$ th time slot of the $k$ th transmission block and $f_{r}^{(k)}$ and $g_{r}^{(k)}$ stand for the channel from $\mathcal{T}_{1}$ to the $r$ th relay and from $\mathcal{T}_{2}$ to the $r$ th relay, respectively, in the $k$ th block. Note that the noise is modeled as an independent and identically distributed Gaussian random variable with zero mean and variance $\sigma_{n}^{2}$. The relay weights its received signal $y_{\mathcal{R} 1_{r}}^{(k)}$ and $y_{\mathcal{R} 2, r}^{(k)}$ defined in (2) and (3), by a beamforming scaling factor, $w_{3}$ and $w_{4}$, respectively, to form a beam steering towards the destination terminal. The $r$ th relay transmits then the resulting signal

$$
t_{\mathcal{R} 3}^{(k)}=w_{3} y_{\mathcal{R} 1, r}^{(k)}, \quad t_{\mathcal{R} 4}^{(k)}=w_{4} y_{\mathcal{R} 2, r}^{(k)}
$$

in the third and forth time slot of the $k$ th transmission block. In [10,20], it has been shown that the optimum amplify and forward (AF) beamforming factors are given by

$$
w_{3}=c_{3} g_{r}^{*} f_{r}^{*}, \quad w_{4}=c_{4} f_{r}^{*} g_{r}^{*},
$$

where $c_{3}=\sqrt{\frac{P_{\mathcal{R}}}{\left(\sigma^{2}+P_{\mathcal{T}_{1}}\left|f_{r}\right|^{2}\right)\left|f_{r}\right|^{2}\left|g_{r}\right|^{2}}}$ and $c_{4}=\sqrt{\frac{P_{\mathcal{R}}}{\left(\sigma^{2}+P_{\mathcal{T}_{2}}\left|g_{r}\right|^{2}\right)\left|g_{r}\right|^{2}\left|f_{r}\right|^{2}}}$. Note that the optimum relay beamforming factors $w_{3}$ and $w_{4}$ require perfect knowledge of the instantaneous channel coefficients $f_{r}$ and $g_{r}$. Note that channel coefficients can be estimated by sending pilot symbols through the communications between the terminals, however, this estimation process results in a reduced throughput and additional overhead. Another drawback of this process is that more pilot symbols are required if the channel changes rapidly. 


\section{THE DECENTRALIZED NON-COHERENT BEAMFORMING}

Let us consider the case of no CSI available at any transmitting or receiving antenna. To facilitate coherent processing at the relays and the destinations, $\mathcal{T}_{t}$ transmits in the first time slot of the $k$ th block the differentially encoded symbol $x_{\mathcal{T}_{t}}^{(k)}$ to the relays, given by

$$
x_{\mathcal{T}_{t}}^{(k)}=x_{\mathcal{T}_{t}}^{(k-1)} s_{\mathcal{T}_{t}}^{(k)}
$$

In the first two time slots of the $k$ th transmission block, the received signals at the $r$ th relay are given by

$$
\begin{aligned}
& y_{\mathcal{R} 1, r}^{(k)}=\sqrt{P_{\mathcal{T}_{1}}} f_{r}^{(k)} x_{\mathcal{T}_{1}}^{(k)}+n_{\mathcal{R} 1, r}^{(k)}, \\
& y_{\mathcal{R}_{2}, r}^{(k)}=\sqrt{P_{\mathcal{T}_{2}}} g_{r}^{(k)} x_{\mathcal{T}_{2}}^{(k)}+n_{\mathcal{R}_{2}, r}^{(k)}
\end{aligned}
$$

where $n_{\mathcal{R} 1, r}^{(k)}$ and $n_{\mathcal{R}_{2}, r}^{(k)}$ denote the noise at the $r$ th relay in the first and second time slot of the $k$ th transmission block and $f_{r}^{(k)}$ and $g_{r}^{(k)}$ stand for the channel from $\mathcal{T}_{1}$ to the $r$ th relay and from $\mathcal{T}_{2}$ to the $r$ th relay, respectively, in the $k$ th block. Similar to (4), in the third and fourth time slot of the $k$ th block, the $r$ th relay weights its received signals $y_{\mathcal{R}_{1}, r}^{(k)}$ and $y_{\mathcal{R}_{2}, r}^{(k)}$ by weighting factors $w_{3}$ and $w_{4}$ and transmits the resulting signals

$$
t_{\mathcal{R}_{3}, r}^{(k)}=w_{\mathcal{R}_{3}, r}^{(k)} y_{\mathcal{R}_{1}, r}^{(k)}, \quad t_{\mathcal{R}_{4}, r}^{(k)}=w_{\mathcal{R}_{4}, r}^{(k)} y_{\mathcal{R}_{2}, r}^{(k)},
$$

respectively. In the following, let us consider the block fading model, i.e., $f_{r}^{(k-1)}=f_{r}^{(k)}$ and $g_{r}^{(k-1)}=g_{r}^{(k)}$ and consider also the signals received at $\mathcal{T}_{2}$. The received signal at $\mathcal{T}_{1}$ can be recovered correspondingly. During the third time slot, the received signal at $\mathcal{T}_{2}$ is given by

$$
y_{\mathcal{T}_{2}}^{(k)}=\sum_{r=1}^{R} g_{r} t_{\mathcal{R}_{3}, r}^{(k)}=g_{r} w_{\mathcal{R}_{3}, r}^{(k)} y_{\mathcal{R}_{1}, r}^{(k)}
$$

From (5), the optimal value of $w_{\mathcal{R}_{3}, r}, r=1,2, \cdots, R$, which leads to a coherent superposition of the signals from all relays and maximizes the signal to noise ratio (SNR) at both terminals, is expressed as

$$
w_{\mathcal{R}_{3}, r}=c_{\mathcal{R}_{3}, r} g_{r}^{*} f_{r}^{*}=\hat{c}_{\mathcal{R}_{3}, r} e^{j \theta_{\mathcal{R}_{1}, r}^{(k)}}
$$

where $c_{\mathcal{R}_{3}, r}=\sqrt{\frac{P_{r}}{\left(\sigma^{2}+P_{\mathcal{T}_{1}}\left|f_{r}\right|^{2}\right)\left|f_{r}\right|^{2}\left|g_{r}\right|^{2}}}$ and $\hat{c}_{\mathcal{R}_{3}, r}=\sqrt{\frac{P_{r}}{\left(\sigma^{2}+P_{\mathcal{T}_{1}}\left|f_{r}\right|^{2}\right)}}$ are scaling factors to maintain the power constraint and $\theta_{\mathcal{R}_{1}, r}^{(k)}=\frac{f_{r}^{*} g_{r}^{*}}{\left|f_{r}\right|\left|g_{r}\right|}=-\left(\measuredangle f_{r}^{(k)}+\measuredangle g_{r}^{(k)}\right)$. In the proposed strategy, it is assumed that there is no CSI available at any transmitting or receiving antenna. Where as, to ensure coherent reception, the phase rotation $\theta_{\mathcal{R} t, r}^{(k)}$ applied at the $r$ th relay in the $k$ th block must be as close as possible to its optimal value $\theta_{\mathcal{R} t, r}^{\text {opt }}$ defined in (11). In the following, we proposed an efficient encoding strategy performed at the relays in which the phases of their received signals are adjusted and a beam steering towards the destination terminal is formed without requiring CSI. Making use of the received signals at each relay from both terminals, $\theta_{\mathcal{R} 1, r}^{(k)}$ can be expressed as

$$
\theta_{\mathcal{R}_{1}, r}^{(k)}=-\left(\measuredangle y_{\mathcal{R}_{1}, r}^{(k-1)}+\measuredangle y_{\mathcal{R}_{2}, r}^{(k)}\right)
$$

For sufficiently large SNR and from (7) and (8), $\measuredangle y_{\mathcal{R}_{1}, r}^{(k)} \approx \measuredangle f_{r}^{(k)}+\measuredangle x_{\mathcal{R}_{1}, r}^{(k)}$ and $\measuredangle y_{\mathcal{R}_{2}, r}^{(k)} \approx \measuredangle g_{r}^{(k)}+\measuredangle x_{\mathcal{R}_{2}, r}^{(k)}$. In this case, equation (12) can be expressed as

$$
\hat{\theta}_{\mathcal{R}_{1}, r}^{(k)} \approx-\left(\measuredangle f_{r}^{(k-1)}+\measuredangle g_{r}^{(k)}\right)-\left(\measuredangle x_{\mathcal{T}_{1}}^{(k-1)}+\measuredangle x_{\mathcal{T}_{2}}^{(k)}\right)
$$

where the phase term $-\left(\measuredangle x_{\mathcal{T}_{1}}^{(k-1)}+\measuredangle x_{\mathcal{T}_{2}}^{(k)}\right)$ in (13) is a constant. For high SNR, the effect of the noise on the phaseshift becomes insignificant and therefore, $\hat{\theta}_{\mathcal{R}_{1}, r}^{(k)} \approx \theta_{\mathcal{R}_{1}, r}^{(k)}$. Making use of the extended block fading assumption, i.e., the channels do not change over two consecutive transmission blocks, we can use $f_{r}$ and $g_{r}$ to represent $f_{r}^{(k)}$ and $g_{r}^{(k)}$, respectively, where, $g_{r}^{(k)}=g_{r}^{(k-1)}=g_{r}, f_{r}^{(k)}=f_{r}^{(k-1)}=f_{r}$. For sufficiently large SNR, equation (10) can be expressed as

$$
y_{\mathcal{T}_{2}}^{(k)} \approx \sum_{r=1}^{R} \beta_{\mathcal{R}_{1}, r}\left|f_{r}^{(k)}\right|\left|g_{r}^{(k)}\right| e^{\left(-j \measuredangle x_{\mathcal{T}_{2}}^{(k)}\right)} s_{\mathcal{T}_{1}}^{(k)}+w_{\mathcal{T}_{2}}^{(k)}
$$


where

$$
w_{\mathcal{T}_{2}}^{(k)}=\sum_{r=1}^{R} \beta_{\mathcal{R}_{1}, r}\left(g_{r}^{(k)} e^{\left(j \theta_{\mathcal{R} 1, r}^{(k)}\right)} n_{\mathcal{R} 1, r}^{(k)}\right)+n_{\mathcal{T}_{2}}^{(k)} .
$$

In the case that the direct link between the two terminals, $\mathcal{T}_{1}$ and $\mathcal{T}_{2}$, is available, the received signal at terminal $\mathcal{T}_{2}$ during the first transmission phase is given by

$$
y_{\mathcal{T}_{2}, \mathrm{dl}}^{(k)}=\sqrt{P_{\mathcal{T}_{1}}} f_{0} x_{\mathcal{T}_{1}}^{(k)}+n_{\mathcal{T}_{2}}^{(k)}
$$

where $n_{\mathcal{T}_{2}}^{(k)}$ denotes the the receiver noise of the $k$ th block at terminal $\mathcal{T}_{2}$ and $x_{\mathcal{T}_{1}}^{(k)}$ is defined in (6). Making use of the received signal $y_{\mathcal{T}_{2}, \text { dl }}^{(k)}$ defined in (16), the decoder at terminal $\mathcal{T}_{2}$ can be expressed as

$$
\arg \min _{s^{(k)}}\left\{\left\|\frac{y_{\mathcal{T}_{2}}^{(k)}}{e^{\left(-j \measuredangle x_{\mathcal{T}_{2}}^{(k)}\right)}}-\left|\frac{y_{\mathcal{T}_{2}}^{(k-1)}}{e^{\left(-j \measuredangle x_{\mathcal{T}_{2}}^{(k-1)}\right)}}\right| s^{(k)}\right\|^{2}+\left\|y_{\mathcal{T}_{2}, \mathrm{dl}}^{(k)}-y_{\mathcal{T}_{2}, \mathrm{dl}}^{(k-1)} s^{(k)}\right\|^{2}\right\} .
$$

\section{BER PERFORMANCE ANALYSIS}

\subsection{Non-coherent technique}

In this section, the theoretical performance of the proposed differential strategy in terms of BER based on the assumptions in Sec. 2. and assuming no CSI available at any transmitting or receiving antenna is introduced. The general conditional BER expression of coherent and non-coherent techniques for $(R+1)$ independent fading paths between the communicating terminals can be approximated as $[16,18,19]$

$$
P_{b}(\gamma) \approx \frac{1}{2^{2(R+1)} \pi} \int_{-\pi}^{\pi} f(\theta) \exp (-\alpha(\theta) \gamma) d \theta
$$

where

$$
\begin{aligned}
& f(\theta)=\frac{b^{2}}{2 \alpha(\theta)} \sum_{r=1}^{R+1}\left(\begin{array}{c}
2 R+1 \\
R
\end{array}\right)\left(\left(\beta^{-R}-\beta^{R+2}\right) \cos \left(R\left(\theta+\frac{\pi}{2}\right)\right)-\left(\beta^{-R+1}-\beta^{R+1}\right) \cos \left((R+1)\left(\theta+\frac{\pi}{2}\right)\right)\right) \\
& \alpha(\theta)=\frac{b^{2}\left(1+2 \beta \sin (\theta)+\beta^{2}\right)}{2},
\end{aligned}
$$

$\beta=a / b$ is constant, the values of $a$ and $b$ depend on the modulation order, e.g., for 4-PSK, $a=\sqrt{2-\sqrt{2}}$ and $b=\sqrt{2+\sqrt{2}}[18]$, and $\gamma=\gamma_{s}+\sum_{r=1}^{R} \gamma_{r}$ where $\gamma_{s}$ and $\gamma_{r}$ denote the instantaneous SNR for the direct link between $\mathcal{T}_{1}$ and $\mathcal{T}_{2}$ and the link between $\mathcal{T}_{1}$ and $\mathcal{T}_{2}$ via the $r$ th relay, respectively, such that

$$
\begin{aligned}
\gamma_{r} & =\frac{P_{\mathcal{T}_{1}} P_{\mathcal{T}_{2}} P_{r}\left|f_{r}\right|^{2}\left|g_{r}\right|^{2}}{\sigma_{n}^{2}\left(2 P_{\mathcal{T}_{2}} P_{r}\left|g_{r}\right|^{2}+P_{\mathcal{T}_{1}} P_{r}\left|f_{r}\right|^{2}+P_{\mathcal{T}_{1}} P_{\mathcal{T}_{2}} \sigma_{f_{r}}^{2}+P_{\mathcal{T}_{2}} \sigma_{n}^{2}\right)} \\
\gamma_{s} & =\frac{P_{\mathcal{T}_{1}}\left|f_{0}\right|^{2}}{2 \sigma_{n}^{2}} .
\end{aligned}
$$

The average BER is obtained by averaging the conditional BER expression $P_{b}(\gamma)$ given in (18) over the random variables using the moment generation function method $[16,18]$, such that

$$
P_{b} \approx \frac{1}{2^{2(R+1)} \pi} \int_{-\pi}^{\pi} f(\theta) \mathcal{M}_{\gamma_{s}}(\theta) \prod_{r=1}^{R} \mathcal{M}_{\gamma_{r}}(\theta) d \theta
$$

where $\mathcal{M}_{\gamma_{i}}(\theta)$ denotes the moment generation function of the instantaneous $\operatorname{SNR} \gamma_{i}, i \in\{s, 1, \cdots, R\} . \mathcal{M}_{\gamma_{r}}(\theta)$ can be obtained through integration over two exponential random variables $\left|f_{r}\right|^{2}$ and $\left|g_{r}\right|^{2}$, such that

$$
\mathcal{M}_{\gamma_{r}}(\theta)=\frac{1}{1+K_{f}}\left(1+\mathcal{A}(\theta) \int_{0}^{\infty} \frac{\exp \left(-u / \sigma_{g_{r}}^{2}\right)}{u+R_{r}(\theta)} d u\right)
$$


where

$$
\begin{aligned}
\mathcal{A}(\theta) & =\frac{K_{f}}{2 P_{\mathcal{T}_{2}}\left(K_{f}+1\right)} \frac{P_{\mathcal{T}_{1}} P_{\mathcal{T}_{2}} \sigma_{f_{r}}^{2}+P_{\mathcal{T}_{1}} P_{r} \sigma_{f_{r}}^{2}+P_{\mathcal{T}_{2}} \sigma_{n}^{2}}{P_{r}} \frac{1}{\sigma_{g_{r}}^{2}}, \\
R_{r}(\theta) & =\frac{P_{\mathcal{T}_{1}} P_{\mathcal{T}_{2}} \sigma_{f_{r}}^{2}+P_{\mathcal{T}_{1}} P_{r} \sigma_{f_{r}}^{2}+P_{\mathcal{T}_{2}} \sigma_{n}^{2}}{2 P_{r} P_{\mathcal{T}_{2}}\left(1+K_{f}\right)} \\
K_{f} & =K_{f}^{d}, \quad K_{f}^{d}=\frac{\alpha(\theta) P_{\mathcal{T}_{1}} \sigma_{f_{r}}^{2}}{2 \sigma_{n}^{2}} .
\end{aligned}
$$

Similar to (24), to obtain $\mathcal{M}_{\gamma_{s}}(\theta)$, we integrate over the exponential random variable $\left|f_{0}\right|^{2}$ to get

$$
\mathcal{M}_{\gamma_{s}}(\theta)=\frac{1}{1+\frac{\alpha(\theta) P_{\mathcal{T}_{1}} \sigma_{f_{r}}^{2}}{2 \sigma_{n}^{2}}} .
$$

Substituting $\theta=\pi / 2$ into (20), $\alpha(\theta)$ can be bounded as $\alpha(\theta) \leq\left(b^{2}(1+\beta)^{2} / 2\right)$. Hence, the BER is upper bounded by

$$
P_{b} \leq \frac{1}{2^{2(R+1)} \pi} \int_{-\pi}^{\pi} f(\theta) B(\theta) \prod_{r=1}^{R} \frac{1}{1+K_{f}}\left(1+\mathcal{A}(\theta) \int_{0}^{\infty} \frac{\exp \left(-u / \sigma_{g_{r}}^{2}\right)}{u+R_{r, \min }(\theta)} d u\right) d \theta
$$

where

$$
\begin{aligned}
B(\theta) & =\frac{1}{1+K_{0}} \\
K_{0} & =K_{0}^{d} \\
K_{0}^{d} & =\frac{\alpha(\theta) P_{\mathcal{T}_{1}} \sigma_{f_{0}}^{2}}{2 \sigma_{n}^{2}}, \\
R_{r, \min }(\theta) & =\frac{P_{\mathcal{T}_{1}} P_{\mathcal{T}_{2}} \sigma_{f_{r}}^{2}+P_{\mathcal{T}_{1}} P_{r} \sigma_{f_{r}}^{2}+P_{\mathcal{T}_{2}} \sigma_{n}^{2}}{2 P_{r} P_{\mathcal{T}_{2}}}\left(1+\frac{P_{\mathcal{T}_{1}} \sigma_{f_{r}}^{2} b^{2}(1+\beta)^{2}}{4 \sigma_{n}^{2}}\right)^{-1} .
\end{aligned}
$$

In the case that the SNR is large, hence $K_{f}>>1$ and $K_{0}>>1$, we can approximate the terms $1 /\left(1+K_{f}\right)$ in (29) by $1 / K_{f}$ and $1 /\left(1+K_{0}\right)$ in (31) by $1 / K_{0}$. For the sake of simplicity, let us further assume that $\sigma_{f_{0}}=\sigma_{f_{1}}=$ $\cdots=\sigma_{f_{R}}=\sigma_{f}$. In this case, the simple approximation of the BER upper bound can be expressed as

$$
P_{b} \leq\left(\frac{2 \sigma_{n}^{2}}{P_{\mathcal{T}_{1}} \sigma_{f}^{2}}\right)^{(R+1)} Z_{\max }(\theta)
$$

where

$$
Z_{\max }(\theta)=\frac{1}{2^{2(R+1)} \pi} \int_{-\pi}^{\pi} \frac{f(\theta)}{\alpha(\theta)^{(R+1)}}\left(1+\mathcal{A}(\theta) \int_{0}^{\infty} \frac{\exp \left(-u / \sigma_{g_{r}}^{2}\right)}{u+R_{r, \min }(\theta)} d u\right) d \theta
$$

Similar to (29), $\alpha(\theta)$ can be bounded by substituting $\theta=-\pi / 2$ into (20) where $\alpha(\theta) \geq\left(b^{2}(1-\beta)^{2} / 2\right)$. Hence, the BER is lower bounded by

$$
P_{b} \geq \frac{1}{2^{2(R+1)} \pi} \int_{-\pi}^{\pi} f(\theta) B(\theta) \prod_{r=1}^{R} \frac{1}{1+K_{f}}\left(1+\mathcal{A}(\theta) \int_{0}^{\infty} \frac{\exp \left(-u / \sigma_{g_{r}}^{2}\right)}{u+R_{r, \max }(\theta)} d u\right) d \theta
$$

where

$$
R_{r, \text { max }}(\theta)=\frac{P_{\mathcal{T}_{1}} P_{\mathcal{T}_{2}} \sigma_{f_{r}}^{2}+P_{\mathcal{T}_{1}} P_{r} \sigma_{f_{r}}^{2}+P_{\mathcal{T}_{2}} \sigma_{n}^{2}}{2 P_{r} P_{\mathcal{T}_{2}}}\left(1+\frac{P_{\mathcal{T}_{1}} \sigma_{f_{r}}^{2} b^{2}(1-\beta)^{2}}{4 \sigma_{n}^{2}}\right)^{-1}
$$


For sufficiently large SNR and using the same approximations and assumptions as in (34), we obtain the simple approximation of the BER lower bound as

$$
P_{b} \geq\left(\frac{2 \sigma_{n}^{2}}{P_{\mathcal{T}_{1}} \sigma_{f}^{2}}\right)^{(R+1)} Z_{\min }(\theta)
$$

where

$$
Z_{\text {min }}(\theta)=\frac{1}{2^{2(R+1)} \pi} \int_{-\pi}^{\pi} \frac{f(\theta)}{\alpha(\theta)^{(R+1)}}\left(1+\mathcal{A}(\theta) \int_{0}^{\infty} \frac{\exp \left(-u / \sigma_{g_{r}}^{2}\right)}{u+R_{r, \max }(\theta)} d u\right) d \theta
$$

From (34) and (38), $(R+1)$ and $Z(\theta)$ denote the diversity gain and the coding gain, respectively, where it can be observed that the full diversity order $(R+1)$ can be achieved when $R$ relay nodes are used in the network.

\subsection{Coherent technique}

In this section, the theoretical performance of the proposed strategy in terms of BER using the same assumptions as in Sec. 2. and assuming perfect CSI available at all transmitting and receiving antenna is proposed. Similar to Sec. 5.1., the general conditional BER expression $P_{b}(\gamma)$ is given in (18). For the coherent technique, $\gamma^{c}=\gamma_{s}^{c}+\sum_{r=1}^{R} \gamma_{r}^{c}$ with

$$
\gamma_{r}^{c}=\frac{P_{\mathcal{T}_{1}} P_{r}\left|f_{r}\right|^{2}\left|g_{r}\right|^{2}}{\sigma_{n}^{2}\left(P_{r}\left|g_{r}\right|^{2}+P_{\mathcal{T}_{1}} \sigma_{f_{r}}^{2}+\sigma_{n}^{2}\right)}
$$

and $\gamma_{s}^{c}=2 \gamma_{s}$ where $\gamma_{s}$ is defined in (22). After averaging the conditional BER expression $P_{b}(\gamma)$ defined in (18) over the Rayleigh distributed random variables similar as in Sec. 5.1., the average BER $\left(P_{b}\right)$ can be approximated as in (23). Similar to Sec. 5.1., $\mathcal{M}_{\gamma_{s}}(\theta)$, obtained by averaging over the exponential random variable $\left|f_{0}\right|^{2}$, is expressed in (28) and $\mathcal{M}_{\gamma_{r}}(\theta)$, obtained by first averaging over the exponential random variables $\left|f_{r}\right|^{2}$ and then averaging over the exponential random variables $\left|g_{r}\right|^{2}$, is expressed in (24) where $K_{f}=2 K_{f}^{d}, K_{f}^{d}$ is defined in (27),

$$
\mathcal{A}(\theta)=\frac{K_{f}}{\left(K_{f}+2\right)} \frac{P_{\mathcal{T}_{1}} \sigma_{f_{r}}^{2}+\sigma_{n}^{2}}{P_{r}} \frac{1}{\sigma_{g_{r}}^{2}}
$$

and

$$
R_{r}(\theta)=\frac{P_{\mathcal{T}_{1}} \sigma_{f_{r}}^{2}+\sigma_{n}^{2}}{P_{r}\left(1+K_{f}\right)}
$$

Similarly as in Sec. 5.1., the upper bound BER, obtained by substituting $\theta=\pi / 2$ into (20), is defined in (29) where $K_{0}=2 K_{0}^{d}, K_{0}^{d}$ is defined in (32), and

$$
R_{r, \min }(\theta)=\frac{P_{\mathcal{T}_{1}} \sigma_{f_{r}}^{2}+\sigma_{n}^{2}}{P_{r}}\left(1+\frac{P_{\mathcal{T}_{1}} \sigma_{f_{r}}^{2} b^{2}(1+\beta)^{2}}{2 \sigma_{n}^{2}}\right)^{-1}
$$

For sufficiently large SNR and using the same approximations and assumptions as in (34), the simple approximation of the BER upper bound can be expressed as

$$
P_{b} \leq\left(\frac{\sigma_{n}^{2}}{P_{\mathcal{T}_{1}} \sigma_{f_{0}}^{2}}\right)^{(R+1)} Z_{\max }(\theta)
$$

where $Z_{\max }(\theta)$ is defined in (35). The lower bound BER, obtained by substituting $\theta=-\pi / 2$ into (20), is defined in (29) where $K_{0}=2 K_{0}^{d}, K_{0}^{d}$ and $\mathcal{A}(\theta)$ are defined in (32) and (41), respectively, and

$$
R_{r, \text { max }}(\theta)=\frac{P_{\mathcal{T}_{1}} \sigma_{f_{r}}^{2}+\sigma_{n}^{2}}{P_{r}}\left(1+\frac{P_{\mathcal{T}_{1}} \sigma_{f_{r}}^{2} b^{2}(1-\beta)^{2}}{2 \sigma_{n}^{2}}\right)^{-1}
$$


In the case that the SNR is large and using the same approximations and assumptions as in (34), the simple approximation of the BER lower bound can be expressed as

$$
P_{b} \geq\left(\frac{\sigma_{n}^{2}}{P_{\mathcal{T}_{1}} \sigma_{f_{0}}^{2}}\right)^{(R+1)} Z_{\min }(\theta)
$$

where $Z_{\min }(\theta)$ is defined in (39). Similar to Sec. 5.1. and from (44) and (46), it can also be observed that we can achieve the diversity order of $R+1$ when $R$ relay nodes are used in the network.

\section{RESULTS AND ANALYSIS}

In our simulations, we assume a relay network with independent flat Rayleigh fading channels. In Fig. 2, we compare the proposed non-coherent strategy with the four-phase coherent and non-coherent DSTC strategy [12, 13] using the Alamouti [19] and the random unitary code, the optimal coherent distributed transmit beamforming strategy, the strategy proposed in [17], and the two-phase Diff-DSTC strategy for TWRNs [14]. To fairly compare the performance of all strategies, the same total transmitted power $\left(P_{T}=P_{\mathcal{T}_{1}}+P_{\mathcal{T}_{2}}+\sum_{r=1}^{R} P_{r}\right.$, where $P_{\mathcal{T}_{1}}=P_{\mathcal{T}_{2}}=\sum_{r=1}^{R} P_{r}$, $\left.P_{1}=P_{2}=\cdots=P_{R}\right)$ and bit rate are used.

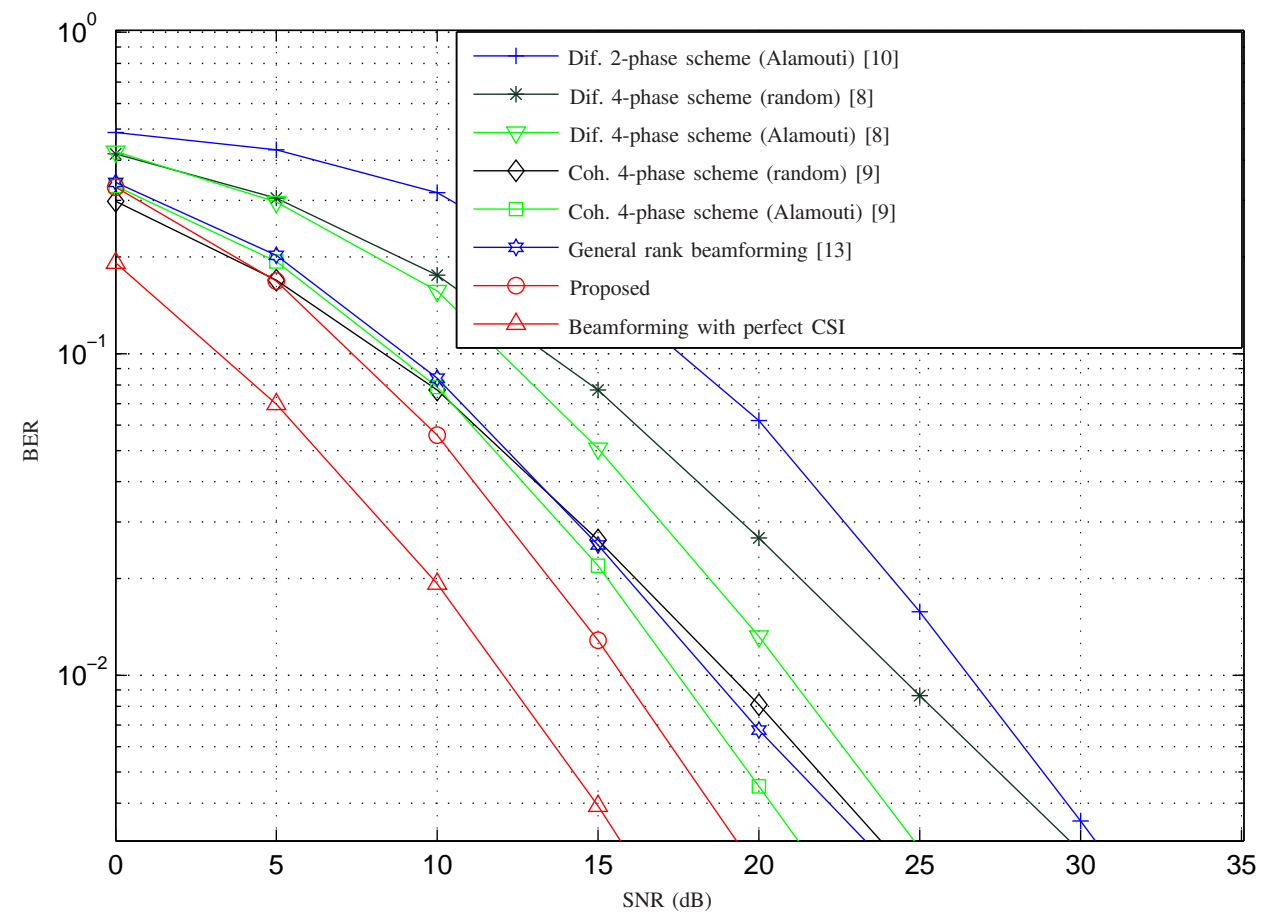

Figure 2. BER versus SNR for different coherent and differential strategies with $R=2$ and a rate of 0.5 bpcu.

It is observed that the proposed strategy outperforms the state-of-the-art two- and four-phase strategies and the difference between the coherent and non-coherent distributed strategy for the random unitary and Alamouti scheme is around $3 \mathrm{~dB}$. Similarly, there exists a $3 \mathrm{~dB}$ margin between the proposed non-coherent strategy and the distributed beamforming strategy. This means that the proposed strategy achieves full diversity gain with coding gain only $3 \mathrm{~dB}$ less than that of a system which requires full CSI at all transmitting and receiving transceivers. The $3 \mathrm{~dB}$ loss in performance can be explained by the absence of CSI at all transmitting and receiving antennas.

In Fig. 3, we consider a relay network with one relay when there is a direct link between the communicating terminals. In this figure, we show the theoretical and simulated BER performance of the proposed strategy at $\mathcal{T}_{2}$ versus the transmitted SNR with and without CSI and using 4-PSK modulation. From Fig. 3, it is observed that the simulated BER performance of our proposed strategy with and without CSI is very close to the theoretical BER performance obtained from the expressions derive in Sec. 5.. 


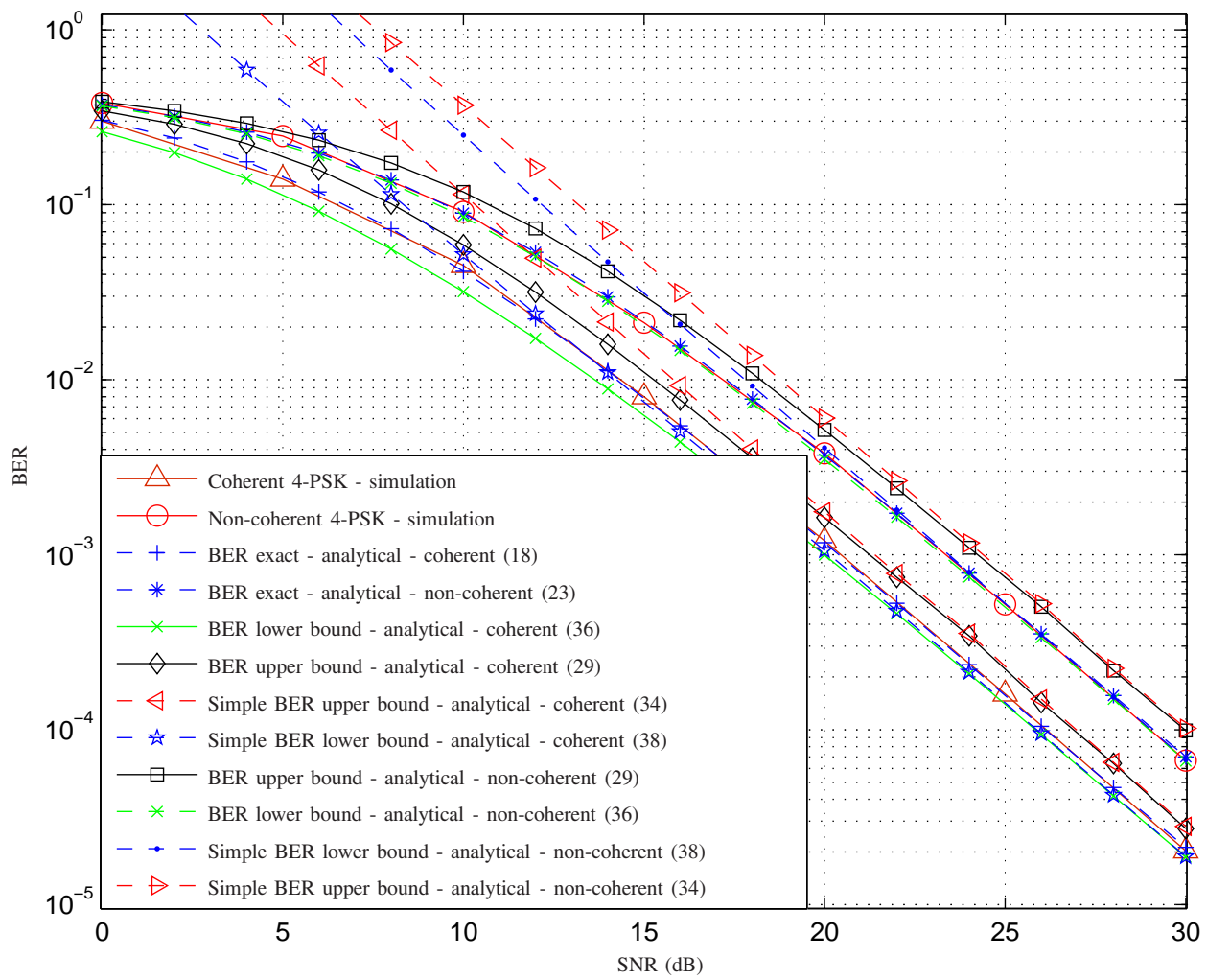

Figure 3. BER versus SNR using 4-PSK.

\section{CONCLUSION}

In this paper, we propose and analyze a novel differential distributed transmit beamforming strategy for decentralized two-way sensor networks. In our proposed strategy, the phases of the received signals at all relay nodes are synchronized without requiring channel feedback or training symbols and with symbol rate equivalent to that of the conventional transmit beamforming strategy. BER expressions of the proposed strategy are provided for coherent and differential M-PSK modulation. Upper bounds, lower bounds, and simple approximations of the BER are also derived. The simple approximation of the BER upper bound shows that the proposed strategy enjoys the full diversity gain which is equal to the number of transmitting antennas.

\section{REFERENCES}

[1] S. Alabed, M. Pesavento, and A. Klein, "Relay selection based space-time coding for two-way wireless relay networks using digital network coding," In Proceedings of the Tenth International Symposium on Wireless Communication Systems, Ilmenau, TU Ilmenau, Germany, Aug. 27-30, 2013.

[2] M. Anusha, et al., "Transmission protocols in Cognitive Radio Mesh Networks," International Journal of Electrical and Computer Engineering, vol. 5, pp. 1446-1451, 2015.

[3] I. B. Oluwafemi, "Hybrid concatenated coding scheme for MIMO systems," International Journal of Electrical and Computer Engineering, vol/issue: 5(3), pp. 464-476, 2015.

[4] Nasaruddin, et al., "Optimized power allocation for cooperative amplify-and-forward with convolutional codes," TELKOMNIKA Indonesian Journal of Electrical Engineering, vol/issue: 12(8), pp. 6243-6253, 2014.

[5] Kehinde Odeyemi and Erastus Ogunti, "Capacity enhancement for high data rate wireless communication system," International Journal of Electrical and Computer Engineering, vol. 4, no. 5, pp. 800 809, 2014.

[6] S. Alabed, J. Paredes, and A. B. Gershman, "A simple distributed space-time coded strategy for two-way relay channels," IEEE Transactions on Wireless Communications, pp. 1260-1265, vol. 11, no. 4, April, 2012.

[7] S. Alabed, M. Pesavento, and A. Gershman, "Distributed differential space-time coding techniques for twoway wireless relay networks," In Proceedings of the Fourth IEEE International Workshop on Computational Advances in Multi-Sensor Adaptive Processing (CAMSAP 11), pp. 221-224, San Juan, Puerto Rico, December 
2011.

[8] S. Alabed, M. Pesavento, and A. Klein, "Distributed differential space-time coding for two-way relay networks using analog network coding," In Proceedings of the 1st European Signal Processing Conference (EUSIPCO’13), Marrakech, Morocco, Sep. 9-13, 2013.

[9] S. Alabed and M. Pesavento, "A simple distributed differential transmit beamforming technique for two-way wireless relay networks," In the 16th International IEEE/ITG Workshop on Smart Antennas (WSA 2012), pp. 243-247, Dresden, Germany, March 2012.

[10] A. Schad, S. Alabed, H. Degenhardt, and M. Pesavento, "Bi-directional differential beamforming for multiantenna relaying," 40th IEEE International Conference on Acoustics, Speech and Signal Processing, 2015.

[11] S. Alabed, M. Pesavento, and A. Klein, "Non-coherent distributed space-time coding techniques for two-way wireless relay networks," EURASIP Special Issue on Sensor Array Processing, Feb. 2013, DOI: 10.1016/j.sigpro.2012.12.001.

[12] Y. Jing and H. Jafarkhani, "Distributed differential space-time coding in wireless relay networks," IEEE Trans. Commun., vol. 56, no. 7, pp. 1092-1100, Jul. 2008.

[13] Y. Jing and H. Jafarkhani, "Using orthogonal and quasi-orthogonal designs in wireless relay networks," IEEE Trans. Infom. Theory, vol. 53, no. 11, pp. 4106-4118, Nov. 2007.

[14] Z. Utkovski, G. Yammine, and J. Lindner, "A distributed differential space-time coding scheme for two-way wireless relay networks," ISIT 2009, Seoul, Korea, pp. 779-783, Jun. 2009.

[15] S. Alabed, "Performance Analysis of Two-Way DF Relay Selection Techniques," Accepted for publication in Elsevier ICT Express, DOI: 10.1016/j.icte.2016.08.008, August, 2016.

[16] T. Himsoon, W. Siriwongpairat, W. Su, and K. Liu, "Differential modulations for multinode cooperative communications,” IEEE Transactions on Signal Processing, vol. 56, no. 7, July 2008.

[17] V. Havary-Nassab, S. ShahbazPanahi, and A. Grami, "Joint transmit-receive beamforming for multi-antenna relaying schemes," IEEE Transactions on Signal Processing, vol. 58, pp. 4966-4972, Sept. 2009.

[18] M. Simon and M. Aliuini, "A unified approach to the probability of error for noncoherent and differentially coherent modulations over generalized fading channels," IEEE Trans. Commun. vol. 46, no. 12, pp. 1625-1638, Dec. 1998.

[19] S. Alamouti, "A simple transmitter diversity scheme for wireless communications," IEEE J. Select. Areas Commun., vol. 16, pp. 1451-1458, Oct. 1998.

[20] B. Khoshnevis, W. Yu, and R. Adve, "Grassmannian beamforming for MIMO amplify-and-forward relaying," IEEE J. Sel. Areas Commun., vol. 26, no. 8, pp. 1397-1407, Oct. 2008.

\section{BIOGRAPHY OF AUTHOR}

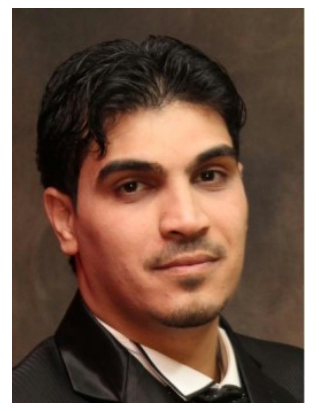

Samer Alabed joined American University of the Middle East as an assistant professor of electrical and computer engineering in 2015. He was a researcher in the communication systems group at Darmstadt University of Technology, Darmstadt, Germany from 2008 to 2015. He received his $\mathrm{PhD}$ degree in electrical engineering and information technology with great honor ("magna cum laude"), from Darmstadt University of Technology, Darmstadt, Germany and his Bachelor and Master degree with great honor. During the last 13 years, he has worked as an assistant professor, (post-doctoral) researcher, and lecturer in several universities in Germany and Middle East where he has taught more than 50 courses in Electrical, Electronic, Communication, and Computer Engineering and supervised tens of master theses and several PhD students. Dr. Alabed received several awards from IEE, IEEE, DAAD ... etc., where the last one was the best paper award from the International IEEE WSA in March, 2015. Dr. Alabed has worked as a researcher in several universities and companies and was invited to many conferences and workshops in Europe, US, and North Africa. The main idea of his research is to develop advanced DSP algorithms in the area of wireless communication systems and networks including (Massive) MIMO systems, distributed systems, co-operative communications, relay networks, space-time block and trellis coding, differential and blind multi-antenna techniques, MIMO channel estimation, MIMO decoders, channel coding and modulation techniques, distributed communication systems, two-way relaying, baseband communications, multi-carrier transmission (OFDM), modeling of wireless channel characteristics, adaptive beamforming, sensor array processing, transceiver design, multi-user and multi-carrier wireless communication systems, convex optimization algorithms for signal processing communications, channel equalization, and other kinds of distortion and interference mitigation.

Further info on his homepage: http://drsameralabed.wixsite.com/samer 